\title{
Sonographic Assessment of a Bifid Median Nerve and Median Artery in Carpal Tunnel Syndrome
}

ANGEL CHECA, MD; HUMAIRA HUSSAIN, MD, Division of Rheumatology, Drexel University College of Medicine, 219 North Broad Street, Philadelphia, Pennsylvania 19107, USA. Address correspondence to Dr. Checa; E-mail: checaag@ comcast.net. J Rheumatol 2011;38:1694-6; doi.10.3899/jrheum.110036

Numerous variants of the median nerve in the carpal tunnel, such as a bifid morphology, have been reported ${ }^{1}$. The prevalence of bifid median nerve with or without a persistent median artery continues to be intriguing. However, a bifid nerve has been observed in patients with carpal tunnel syndrome (CTS). The nerve compression via trauma, dilatation, thrombosis, and arterial calcification is disputed $^{2}$.

An ultrasound scan in longitudinal and transverse planes at the volar aspect of both wrists, at the level of the carpal tunnel, was obtained in 3 patients. Scans were performed

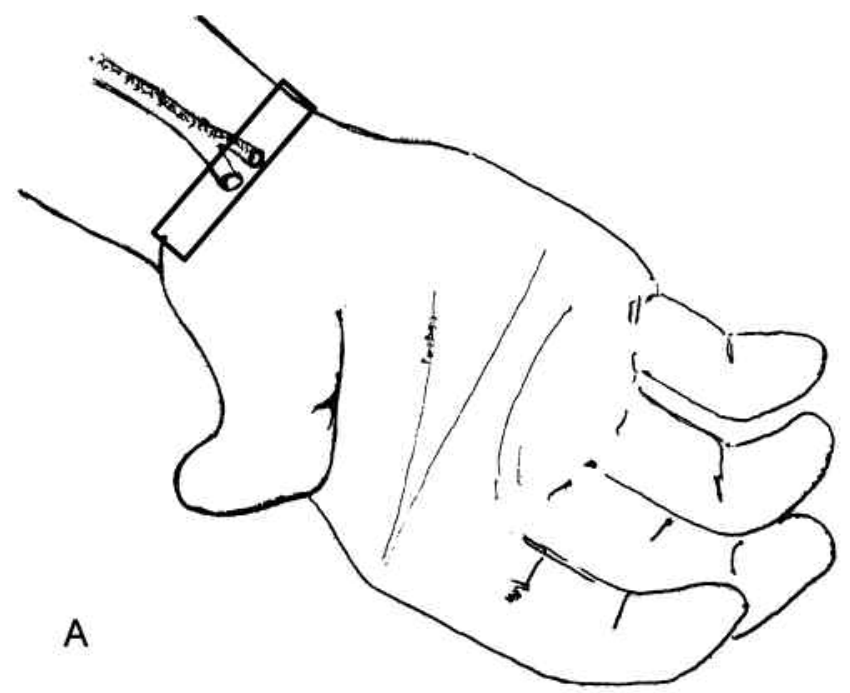

using a GE Logiq e ultrasound machine equipped with a broadband linear probe from 8 to $13 \mathrm{MHz}$.

The first case, an 80-year-old woman with history of osteoarthritis of the hands, presented with tingling and numbness at the median nerve area in the right hand for roughly 1 year. Examination revealed a positive Phalen's test and atrophy of the thenar eminence on the right side. Enlargement of the right median nerve was documented by ultrasound. The left median nerve had a bifid appearance with an accompanying median artery, but it showed normal size (Figure 1).

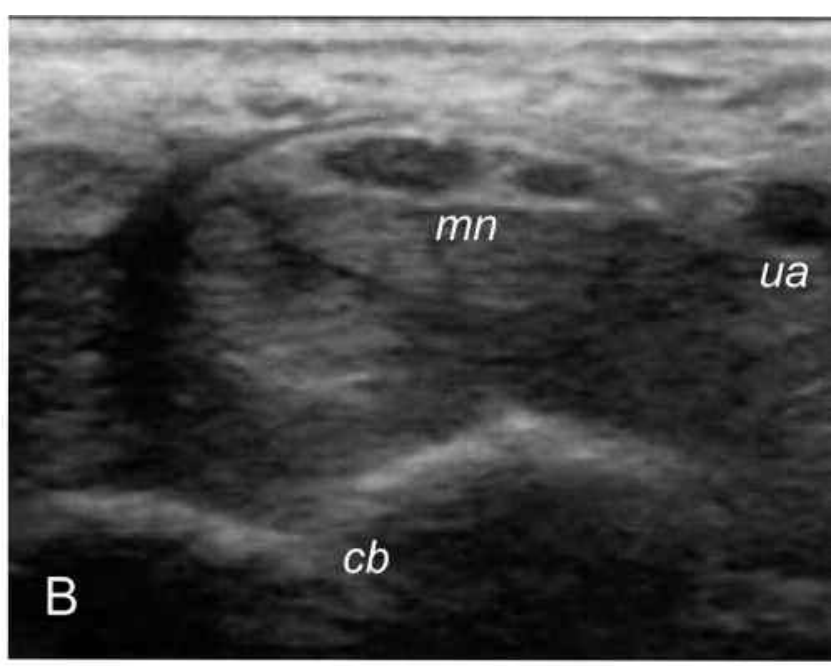

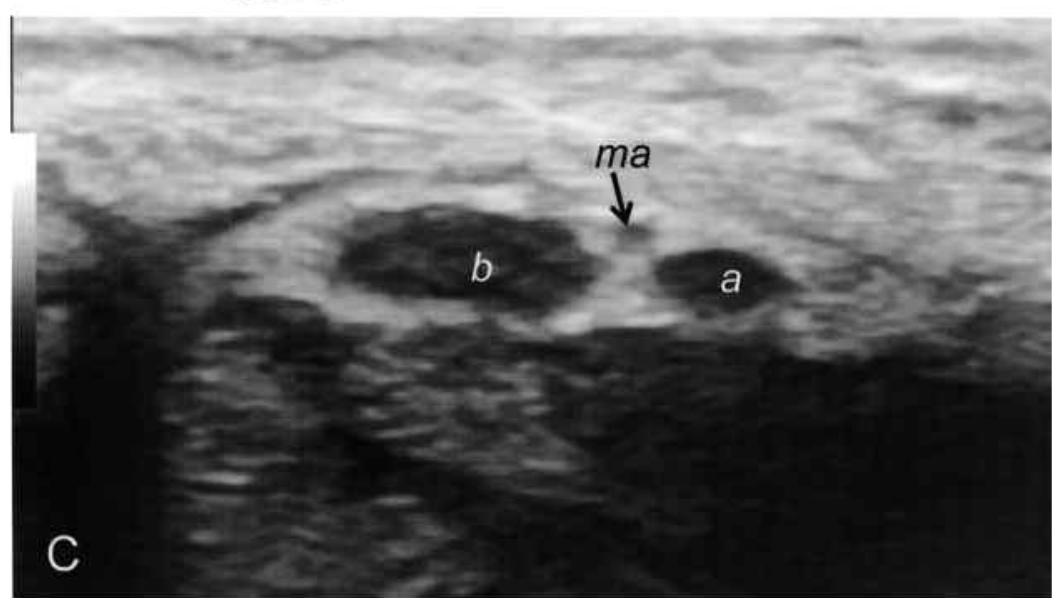

Figure 1. A. The transverse orientation of the transducer. B. Transverse sonographic view of the volar aspect of the wrist reveals a bifid median nerve (mn), carpal bones (cb), and ulnar artery (ua). C. Magnified image reveals medial (a) and lateral (b) branches of bifid nerve, and the median artery (ma). 
The second case was a 55-year-old woman with history of rheumatoid arthritis (RA) and bilateral symptoms consistent with CTS for several months. A positive Phalen's test and atrophy of the thenar eminence was observed bilaterally. Sonographic evaluation of both median nerves revealed bilateral enlargement with a bifid morphology on the left side. Medial and lateral branches emerging from a common trunk were also documented by ultrasound (Figure 2).

The third case was a 52-year-old woman, also with his- tory of RA, who had bilateral numbness in the area of the median nerve with a positive Phalen's test and atrophy of the thenar eminence in both hands. Sonographic evaluation of the median nerve showed bilateral enlargement with a bifid morphology in the left wrist. Cross-sectional area was measured separately in each branch. A total value for both branches was $14 \mathrm{~mm}^{2}$ (Figure 3), and the lateral branch was found to be almost twice the size of the medial branch.

The prevalence of bifid median nerve and median artery
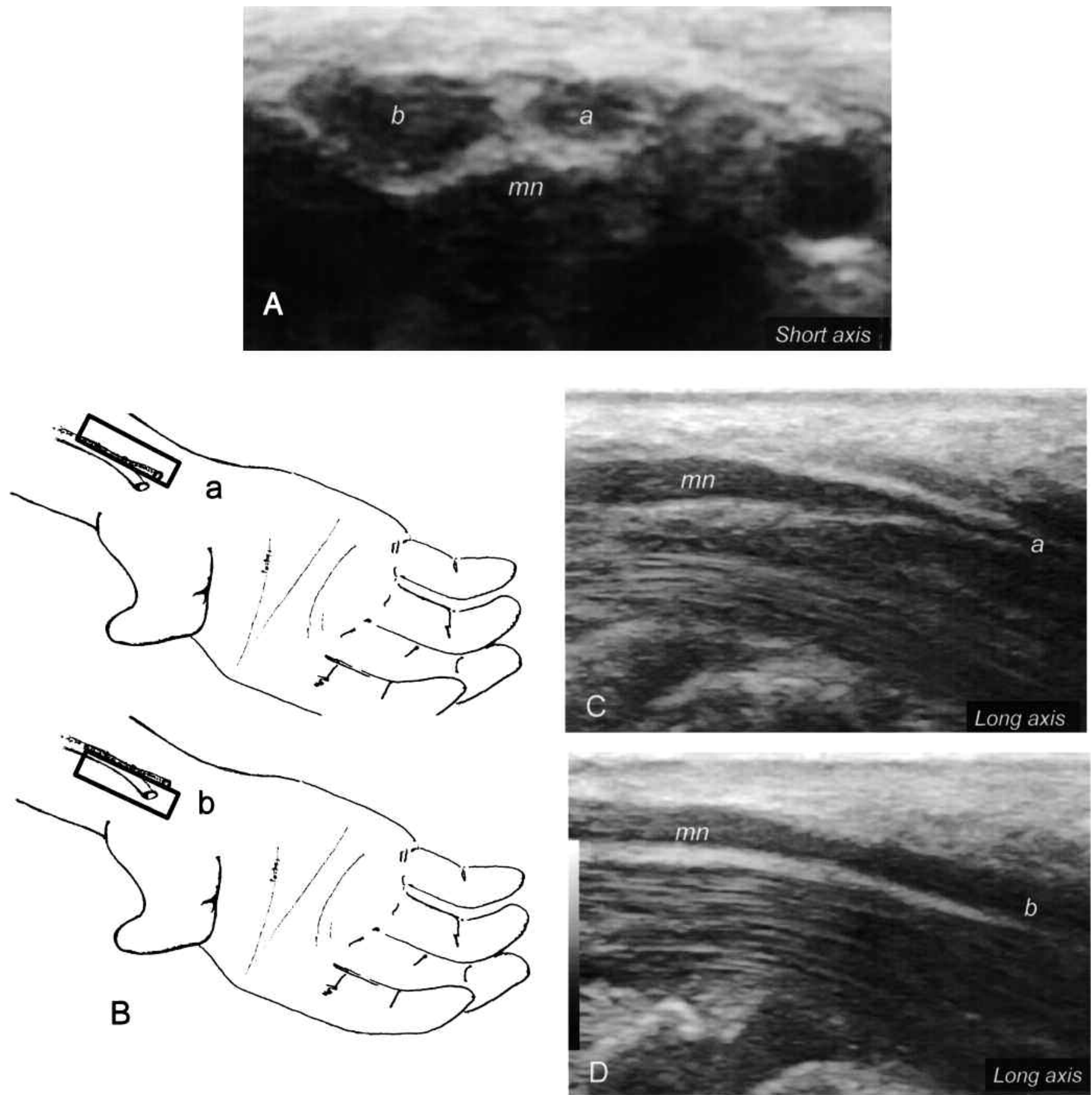

Figure 2. A. Bifid median nerve without median artery. B. Orientation of a longitudinal sonographic scan for examination of the medial (a) and lateral (b) branches emerging from a common trunk; these are shown sonographically (c and d). 


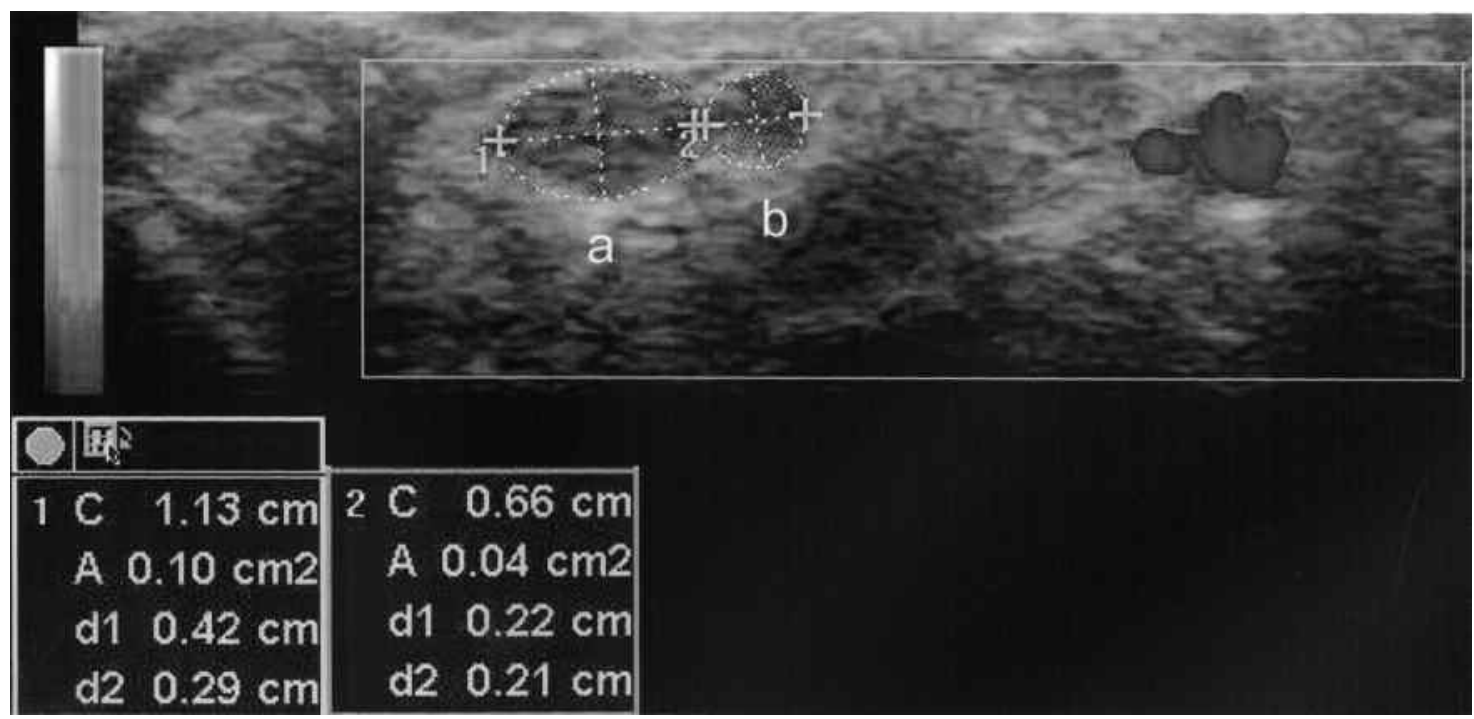

Figure 3. Cross-sectional sonographic measurement of lateral (a) and medial branch (b) of the bifid median nerve in a 52-year-old woman with manifestations of CTS.

and other anomalies in the carpal tunnel remains unclear. Most reports are based on surgical explorations and symptomatic subjects. These anomalies appear to be discovered more frequently with use of modern techniques such as magnetic resonance imaging (MRI) and high frequency ultrasonography $y^{3,4,5}$. Pierre, et $a l^{5}$, in a study of 194 MRI scans of the wrist at the carpal tunnel level, found rates as high as $18 \%$ for bifid median nerve and $11 \%$ for median artery.

The appropriate way to calculate the size of the bifid median nerve is not amply described. We considered that enlargement was present in those cases in which the cross-sectional area of both branches at the scaphoid-pisiform level with the ellipse was greater than $10 \mathrm{~mm}^{2}$. In non-bifid nerve this cutoff value has been considered indicative of CTS by some investigators ${ }^{6}$.

The increased observation of bifid median nerve by ultrasound, a tool with increasing use in rheumatology, should be considered an alert to pay more attention to different variants of the median nerve at the carpal tunnel level, rather than considering the phenomenon purely as a medical curiosity. Identification of a bifid median nerve and median artery with noninvasive and inexpensive ultrasound imaging technology may avoid serious complications such as laceration of the artery during injection or in the operating theater.

\section{REFERENCES}

1. Berry MG, Vijh V, Percival NJ. Bifid median nerve: Anatomical variant at the carpal tunnel. Scand J Plast Reconstr Surg Hand 2003;37:58-60.

2. Sañudo JR, Chikwe J, Evans SE. Anomalous median nerve associated with persistent median artery. J Anat 1994;185:447-51.

3. Propeck T, Quinn TJ, Jacobson JA, Paulino AFG, Habra G, Darian VG. Sonography and MR imaging of bifid median nerve with anatomic and histologic correlation. AJR Am J Roentgenol 2000;175:1721-5

4. Iannicelli E, Chianta GA, Salvini V, Almberger M, Monacelli G, Passariello R. Evaluation of bifid median nerve with sonographic and MR imaging. J Ultrasound Med 2000;19:481-5.

5. Pierre-Jerome C, Smitson RD Jr, Shah RK, Moncayo V, Abdelnoor M, Terk MR. MRI of the median nerve and median artery in the carpal tunnel: prevalence of their anatomical variations and clinical significance. Surg Radiol Anat 2010;32:315-22.

6. Klauser AS, Halpern EJ, De Zordo T, Feuchtner GM, Arora R, Gruber J, et al. Carpal tunnel syndrome assessment with US: value of additional cross-sectional area measurements of the median nerve in patients versus healthy volunteers. Radiology 2009;250:171-7. 